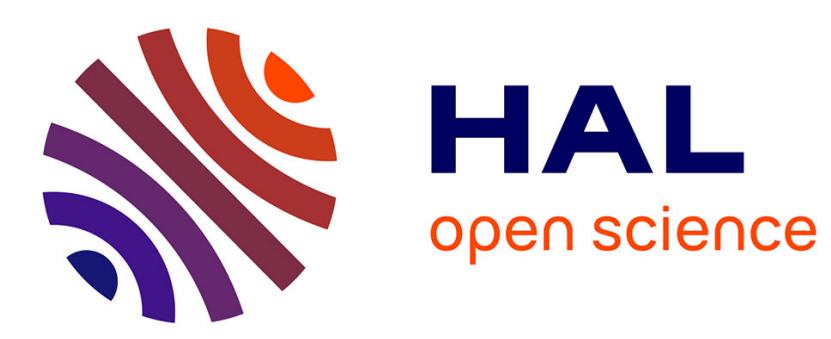

\title{
Impact of Propagation Losses on Fault Location Accuracy in Full Transient-Based Methods
}

\author{
Andréa Cozza, Shao-Yin He, Yan-Zhao Xie
}

\section{To cite this version:}

Andréa Cozza, Shao-Yin He, Yan-Zhao Xie. Impact of Propagation Losses on Fault Location Accuracy in Full Transient-Based Methods. IEEE Transactions on Power Delivery, 2020, 10.1109/tpwrd.2020.2983323 . hal-02515909

\section{HAL Id: hal-02515909 \\ https://hal.science/hal-02515909}

Submitted on 23 Mar 2020

HAL is a multi-disciplinary open access archive for the deposit and dissemination of scientific research documents, whether they are published or not. The documents may come from teaching and research institutions in France or abroad, or from public or private research centers.
L'archive ouverte pluridisciplinaire HAL, est destinée au dépôt et à la diffusion de documents scientifiques de niveau recherche, publiés ou non, émanant des établissements d'enseignement et de recherche français ou étrangers, des laboratoires publics ou privés. 


\title{
Impact of Propagation Losses on Fault Location Accuracy in Full Transient-Based Methods
}

\author{
Andrea Cozza, Senior Member, IEEE, Shao-yin He, Member, IEEE, Yan-zhao Xie, Senior Member, IEEE
}

\begin{abstract}
This paper studies how propagation and termination losses affect full transient-based fault location techniques. Their accuracy is discussed in terms of both location uncertainty, caused by a limited spatial resolution, and systematic errors, caused by a bias in the fault-location metrics. This last case is proven to be by far likelier when propagation losses are higher than the dissipation in line termination loads. Two different location metrics are studied, namely correlation and normalized projection, as found in the literature, with correlation proven to be unbiased, since it benefits from two location mechanisms, namely frequency and time-decay matching of a line resonances, as opposed to projection, which only relies on the former mechanism. A numerical analysis of realistic lossy overhead lines confirms theoretical predictions about biased fault location and loss of spatial resolution and the role played by the frequency content of transient data. When applied to the modal analysis of a three-phase transmission line, these results help explaining why faults are located with widely variable accuracy depending on their distance and the bandwidth of the recorded transient, confirming that wide-band transient sampling does not necessarily results in the best location accuracy.
\end{abstract}

Index Terms-Fault location, transmission lines, location accuracy, propagation losses, modal theory.

\section{INTRODUCTION}

$\mathbf{T}$ HE location of faults such as phase-to-ground shunt connections can be estimated from the transients they generate, recorded by one or multiple probes monitoring the ends of a line [1], [2]. Traveling-wave methods (TWM) use the early-time portion of these transient signals in order to estimate a fault position, relying on differences in the time of arrival of successive echoes of a fault-initiated surge. A number of variants have been developed during the last decades, mainly divided into single- and multiple-ended methods [3]-[11].

As opposed to TWM, an alternative group of location methods has more recently been developed, using the entirety of transients recorded by a single probe. They can therefore be referred to as full transient-based methods (TBM) and share the idea of locating a fault by measuring the similarity between measured and reference (typically simulated) transients signals [12]-[21]. TBM have been shown to present some advantages compared to TWM, as discussed at the end of [13]. Among them, the ability of TBM to provide accurate fault location

(Corresponding author: A. Cozza.).

S.-Y. He, Y.-Z. Xie, are with the State Key Laboratory of Electrical Insulation and Power Equipment, School of Electrical Engineering, Xi'an Jiaotong University, Xi'an 710049, China (email: shaoyin.he@xjtu.edu.cn; yzxie@xjtu.edu.cn).

A. Cozza is with the Group of Electrical Engineering - Paris (GeePs), CentraleSupelec, Univ. Paris-Sud, Université Paris-Saclay, Sorbonne Universites, UPMC Univ Paris 06, 3 \& 11 rue Joliot-Curie, Plateau de Moulon 91192 Gif-sur-Yvette CEDEX, France (email: andrea.cozza@ieee.org).
[22], [23] without relying on high-speed sampling, while monitoring transients from a single end of a line.

The mechanisms underpinning their high location accuracy were theoretically explained in [24], firmly linking spatial resolution to the degree of resonance of a transmission line subject to a shunt fault. As such, propagation losses should be expected to have a significant impact on fault location accuracy, since they act as a damping mechanism, thus limiting a line's resonances.

The main drawback of TBM is the need for a collection of reference transients corresponding to tested, or candidate, fault positions. The results of TBM are usually presented as if location metrics should be expected to reach their maximum value at a single position close to the fault, but to the best of our knowledge this has not been proven to be a general property. Furthermore, the effects of losses on fault location do not appear to have been formally studied, making it difficult to understand and predict how they affect fault location accuracy. Results of a limited comparative analysis on the effects of propagation losses were discussed in [25], showing that they may indeed lead to erroneous fault locations.

One of the goals of this paper is to explain what mechanisms in TBM enable an accurate fault location and under what conditions erroneous fault positions may occur. The paper especially focuses on the role played by losses in this respect, showing that even in case of relatively weak propagation losses it is possible to observe a bias, with faults inaccurately located much closer to the probe than in reality.

The paper starts by discussing in Sec. II the two similarity metrics found throughout the literature, namely projection and correlation, based on full-transient recording. The problem of location ambiguity is addressed in Sec. IV, by studying the location mechanisms of transient similarity, based on the line resonance models summarized in Sec. III. Significant differences in the accuracy of correlation- and projectionbased location are pointed out, depending on propagation and termination losses. Sec. V argues about the importance of compensating a fault surge spectrum, in order to avoid a low-frequency bias, that would systematically hinder accurate location properties. Numerical results for a single-phase lossy overhead line in Sec. VI support theoretical predictions, among which the risk of ambiguous location when using projection rather than correlation metrics and the marginal benefits of extending tests over frequencies above a few tens of $\mathrm{kHz}$. These conclusions are extended in VII to a three-phase line, where a modal analysis helps explaining why the accuracy of a fault location may significantly differ depending on the fault distance and the bandwidth over which its transient is 


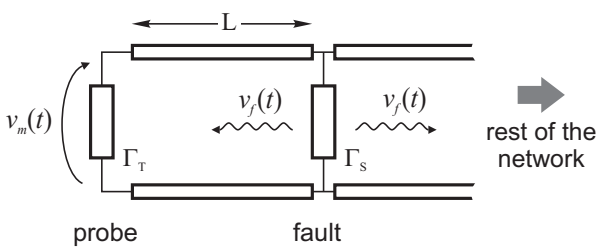

Fig. 1. Single-line model considered in the theoretical analysis. $\Gamma_{T}$ and $\Gamma_{S}$ represent the reflection coefficients of the transformer and the shunt fault, respectively.

recorded. These results have direct practical implications in the improvement of TBM fault location techniques, by providing a general framework for understanding the conditions warranting accurate results, in particular for distant faults.

\section{MEASURING SIMILARITY}

The scenario studied throughout this paper is represented in Fig. 1, based on a uniform single-phase transmission line, terminated at its left end by a power transformer, where a probe monitors the appearance of transient signals.

TBM work by measuring the similarity between the measured transient generated by a fault at a distance $L$, e.g., the voltage ${ }^{1} v_{m}(t ; L)$ measured by a probe $^{2}$, and reference transients $v_{m}(t ; \hat{L})$, obtained either by means of numerical simulations or collections of experimental data, corresponding to potential faults at a set of distances $\hat{L}$. Their similarity can be measured from their projection

$$
\mathcal{P}(L, \hat{L})=\left[v_{m}(t ; L) \mid v_{m}(t ; \hat{L})\right]=\int \mathrm{d} t v_{m}(t ; L) v_{m}(t ; \hat{L}),
$$

with brackets used as a short-hand notation. As done in previous work about similarity [12]-[14], both measured and reference transient signals will be assumed to be synchronized to their rising fronts, e.g., thanks to transient-based triggering.

The results of projection need to be normalized with respect to a reference, in order to offer a quantitative reading. Two choices are found in the literature and compared in this paper. An a posteriori normalization with respect to the maximum value of the projection, i.e., a global normalization

$$
\mathcal{P}_{n}(L, \hat{L})=\frac{\mathcal{P}(L, \hat{L})}{\max _{x}|\mathcal{P}(L, x)|},
$$

is found in electromagnetic time-reversal (EMTR) methods [15], [16]. A local normalization

$$
\rho(L, \hat{L})=\frac{\mathcal{P}(L, \hat{L})}{\sqrt{\mathcal{P}(L, L) \mathcal{P}(\hat{L}, \hat{L})}}
$$

is applied in correlation-based methods [12]-[14], i.e., adopting a position-dependent paradigm. Assuming transients to have a negligible average value, (3) corresponds to the correlation coefficient. Correlation metrics can also be applied to EMTR, as shown in [16].

\footnotetext{
${ }^{1}$ alternatively, transient currents could also be monitored.

${ }^{2}$ assumed to present a linear response, potentially frequency dependent, allowing to convert its output signal into the transient that originated it.
}

Fault positions are then estimated as the set of positions $\hat{L}$ yielding maximum similarity, which could include more than one viable position.

\section{FAULT-TRANSIENT THEORY FOR A SINGLE-PHASE LINE}

The accuracy of fault location metrics (2) and (3) is first studied for single-phase lines in Secs. IV and VI. The rationale for this choice is twofold: first, single-phase line models have a practical importance of their own, as they are represent widely used lines such as in low-voltage distribution networks, monopole high-voltage DC lines and high-voltage coaxial cables used both in underground and submarine lines [26, Sec. 5]; second, their afford simple yet detailed models for which general conclusions can be drawn. These will be shown to apply also to the more complex case of three-phase lines, where a modal analysis is necessary, as shown in Sec. VII.

A model of transient generation and its relationship with a fault's location can be derived based on transmission-line theory [27]. With reference to Fig. 1, a shunt fault is found at a distance $L$, assumed to have a significantly lower impedance than the characteristic impedance of the line, thus practically severing the line into two parts: for the sake of simplicity, the line will be approximated to have a length $L$, with negligible energy transmitted across the fault. The fault is modelled as generating a surge (or fault) signal $v_{f}(t)$, which will afterward travel along the two sections of the line. The fault surge is described as a step function, with a finite rise time $T_{r}$ to reach its maximum value $V_{o}$

$$
v_{f}(t)=V_{o}\left(1-\mathrm{e}^{-t / T_{r}}\right)
$$

and a Fourier spectrum

$$
V_{f}(\omega)=\frac{V_{o} / T_{r}}{\mathrm{j} \omega\left(\mathrm{j} \omega+1 / T_{r}\right)},
$$

where $\omega$ is the angular frequency ${ }^{3}$.

The interactions of the fault surge signal with the line terminations are modelled by means of equivalent reflection coefficients, $\Gamma_{T}=\left|\Gamma_{T}\right| \exp \left(\mathrm{j} \varphi_{T}\right)$ for the transformer and $\Gamma_{S}=\left|\Gamma_{S}\right| \exp \left(\mathrm{j} \varphi_{S}\right)$ for the shunt fault. Both can be expected to have a modulus close to one, with the transformer presenting an equivalent input impedance much higher than the characteristic impedance of the line [1], [3]. Transmission-line theory states that the transient voltage $v_{m}(t ; L)$ measured by the probe at the left end of the line in Fig. 1 has a Fourier spectrum [27]

$$
V_{m}(\omega ; L)=V_{f}(\omega)\left(1+\Gamma_{T}\right) \mathrm{e}^{-\gamma L} H(\omega ; L),
$$

with

$$
H(\omega ; L)=\left(1-\Gamma_{T} \Gamma_{S} \mathrm{e}^{-2 \gamma L}\right)^{-1} .
$$

Neglecting the contribution from $H(\omega)$, (6) represents the direct-path transfer function between fault and probe, where $\gamma(\omega)=\alpha(\omega)+\mathrm{j} \beta(\omega)$, with $\beta(\omega)=\omega / v(\omega)$ the propagation constant, $v(\omega)$ the propagation speed and $\alpha(\omega)$ the line attenuation constant. As recalled in the previous section, both

\footnotetext{
${ }^{3}$ later dropped for the sake of compactness
} 
measured and reference transient signals are assumed to be synchronized to their rising fronts, reducing (6) to

$$
V_{m}(\omega ; L)=V_{f}(\omega)\left(1+\Gamma_{T}\right) \mathrm{e}^{-\alpha L} H(\omega ; L) .
$$

The function $H(\omega)$ represents the resonant response of the portion of line between fault and probe, accounting for its closed-loop behavior, i.e., multiple reflections over its terminations. $H(\omega)$ has an infinite set of conjugate complex poles, giving rise to individual responses $\psi\left(\omega ; \omega_{m}\right)$,

$$
H(\omega)=\sum_{m=1}^{\infty} \frac{2}{T_{m}} \frac{\mathrm{j} \omega+1 / \tau_{m}}{\left(\mathrm{j} \omega+1 / \tau_{m}\right)^{2}+\omega_{m}^{2}}=\sum_{m=1}^{\infty} \frac{2}{T_{m}} \psi\left(\omega ; \omega_{m}\right)
$$

with

$$
\begin{aligned}
\omega_{m} & =\left(2 m \pi+\varphi_{S}+\varphi_{T}\right) / T_{m} \\
\tau_{m} & =\frac{T_{m}}{2 \alpha L-\ln \Gamma}
\end{aligned}
$$

the angular frequency and decay time of its $m$-th order resonance, respectively, with $\Gamma=\left|\Gamma_{T} \Gamma_{S}\right|$ and $T_{m}=2 L / v\left(\omega_{m}\right)$ the round-trip delay time along the line [24]. In (10b), the term $2 \alpha L$ models propagation losses, while $-\ln \Gamma$ models dissipation in the line termination impedances. Although both have an equivalent effect on (10b), Section IV will show that depending on their ratio the location accuracy can be significantly affected.

For the line to sustain resonances, and therefore enable accurate fault location, it is necessary that $\tau_{m} \gtrsim T_{m}$, hence $2 \alpha L-\ln \Gamma \lesssim 1$. Under these conditions, the spatial resolution $D_{c}$ afforded by a single resonance was shown in [24] to directly depend on losses as

$$
D_{c}=\lambda_{m} \frac{2 \alpha L-\ln \Gamma}{2 \pi},
$$

where $\lambda_{m}$ is the wavelength associated to the $m$-th resonance.

Of practical importance for later discussions is the case of dominant propagation losses, i.e., for $2 \alpha L \gg-\ln \Gamma$, for which the decay time

$$
\tau(\omega) \simeq 1 / \alpha(\omega) v(\omega)
$$

no longer depends on the line length, i.e., the fault distance.

\section{LOCATION AMBIGUITY:SINGLE-RESONANCE ANALYSIS}

The possibility of location ambiguity when using similarity metrics is here discussed. The analysis will be restricted to the case of data covering only a single resonance of the line. The rationale for this choice was established in [24] by showing that the location properties of projection metrics are determined by the mismatch between the individual resonances of the measured and reference fault transients. These resonances are here proven to also contain the seeds of inaccurate fault location. These results will serve as guidelines in the interpretation of projection and correlation fault location metrics in the more general conditions discussed in Secs. VI and VII.

Systematic location errors can be identified by means of the location ambiguity function

$$
\mathcal{A}_{p}(L, \hat{L})=\left|\frac{\mathcal{P}_{n}(L, \hat{L})}{\mathcal{P}_{n}(L, L)}\right|=\left|\frac{\mathcal{P}(L, \hat{L})}{\mathcal{P}(L, L)}\right|,
$$

here defined for the projection metric (2). Ideally, $\mathcal{A}_{p}(L, \hat{L})<$ $1, \forall \hat{L} \neq L$, with the fault position unambiguously identified by the global maximum of $\mathcal{P}_{n}(L)$. Two non-ideal cases may occur in practice: 1) if $\mathcal{A}_{p}(L, \hat{L})=1$ also for values of $\hat{L} \neq L$, the interpretation of the location metric would become ambiguous, with multiple candidate positions where $\mathcal{P}_{n}$ reaches the same peak amplitude; 2) a worse condition is encountered if $\mathcal{A}_{p}(L, \hat{L})>1$, since in this case the actual fault position would not even be considered as a viable candidate, because $\mathcal{P}_{n}(\hat{L})>\mathcal{P}_{n}(L)$ for $\hat{L} \neq L$.

\section{A. Projection}

The projection (1) can be transposed in the frequency domain thanks to Parseval theorem, applying it to (8) for a narrow bandwidth centered at $\omega_{o}$, yielding

$$
\mathcal{P}(L, \hat{L}) \simeq \mathrm{e}^{-\alpha(L+\hat{L})}\left|V_{f}\left(\omega_{o}\right)\right|^{2} \int \mathrm{d} \omega H^{\star}(\omega ; L) H(\omega ; \hat{L}),
$$

where the term $\left(1+\Gamma_{T}\right)^{2}$ is dropped for compactness, having no bearing on the following derivation.

Over the tested bandwidth the actual and reference lines will be assumed to present a single resonance at $\omega_{p}$ and $\hat{\omega}_{p}$, respectively, each associated to a decay time $\tau$ and $\hat{\tau}$, as defined in (10). Close resonant frequencies ${ }^{4}$ can be found also in case $\hat{L} \neq L$, as implicit in (10a), a potential cause of ambiguity discussed in Sec. VI.

From (9)

$$
\mathcal{P}(L, \hat{L}) \simeq 4 \frac{\mathrm{e}^{-\alpha(L+\hat{L})}}{T \hat{T}}\left|V_{f}\left(\omega_{o}\right)\right|^{2}\left[\psi\left(\omega ; \omega_{p}\right) \mid \psi\left(\omega ; \hat{\omega}_{p}\right)\right],
$$

with $T=2 L / v\left(\omega_{o}\right)$ and $\hat{T}=2 \hat{L} / v\left(\omega_{o}\right)$, assuming $v\left(\omega_{p}\right), v\left(\hat{\omega}_{p}\right) \simeq v\left(\omega_{o}\right)$.

The projection is more easily computed in the time domain, based on the time-domain resonance response $\psi\left(t ; \omega_{p}\right)=$ $\exp (-t / \tau) \cos \left(\omega_{p} t\right)$. Hence,

$$
\begin{aligned}
{\left[\psi\left(\omega ; \omega_{p}\right) \mid \psi\left(\omega ; \hat{\omega}_{p}\right)\right] } & =\int \mathrm{d} t \mathrm{e}^{-t / \bar{\tau}} \cos \left(\omega_{p} t\right) \cos \left(\hat{\omega}_{p} t\right) \\
& \simeq \frac{\bar{\tau} / 2}{1+(2 \pi \Delta f \bar{\tau})^{2}}
\end{aligned}
$$

where $\Delta f=\left(\omega_{p}-\hat{\omega}_{p}\right) / 2 \pi$ is the frequency mismatch between the resonances of the actual and reference lines and

$$
\bar{\tau}=\left(\tau^{-1}+\hat{\tau}^{-1}\right)^{-1}=\frac{\tau \hat{\tau}}{\tau+\hat{\tau}} .
$$

The resonance mismatch $\Delta f$ is better appreciated if expressed in terms of the coherence bandwidth of the actual line, $B_{c}=$ $1 / \pi \tau$, which measures the spectral selectivity (or resolution) of a single resonance [24]. Hence,

$$
2 \pi \Delta f \bar{\tau}=\eta \Delta f / B_{c}
$$

with

$$
\eta=2 \frac{\bar{\tau}}{\tau}=\frac{2 \hat{\tau} / \tau}{1+\hat{\tau} / \tau}
$$

where $\eta \in[0,2]$ and $\eta=1$ for $\hat{\tau}=\tau$.

\footnotetext{
${ }^{4}$ but of different order
} 

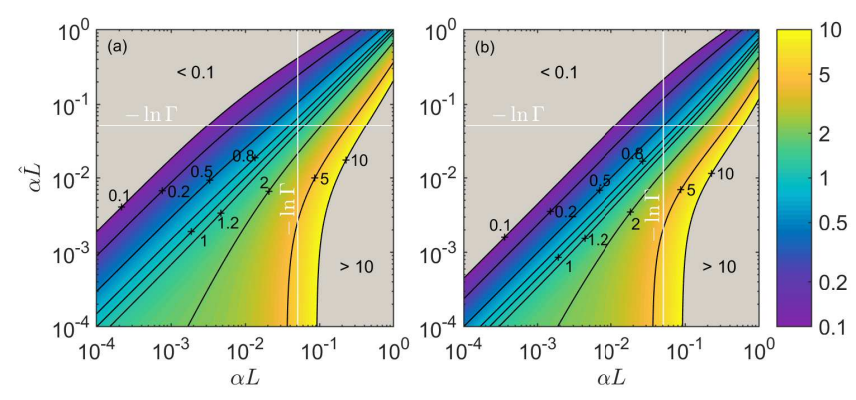

Fig. 2. Location ambiguity function $\mathcal{A}_{p}(L, \hat{L})$ for the projection, for $\Gamma=\left|\Gamma_{S} \Gamma_{T}\right|=0.95$, evaluated from (20) for (a) matched resonances, i.e., $\Delta f / B_{c}=0$ and (b) $\Delta f / B_{c}=1$ for a single resonance. Only results within the range $[0.1,10]$ are shown.

The location ambiguity function (13) can now be computed from (15)-(19), yielding

$$
\mathcal{A}_{p}(L, \hat{L}) \simeq \eta \frac{L}{\hat{L}} \frac{\mathrm{e}^{\alpha(L-\hat{L})}}{1+\left(\eta \Delta f / B_{c}\right)^{2}}
$$

and is shown in Fig. 2. The term $\eta \Delta f / B_{c}$ in the denominator effectively reduces the projection, and therefore the ambiguity, as soon as a resonance mismatch exists between the actual and tested line responses. But this property alone is not sufficient to guarantee that the exact fault position is identified, since a resonance match can be observed also for $\hat{L} \neq L$.

Necessary conditions for systematic location errors are found from (20) by requiring $\mathcal{A}_{p}(L, \hat{L})>1$, thus for a) $\eta L / \hat{L}>1$, i.e., testing positions closer to the probe than the actual fault or b) $\exp (\alpha L)>1$, which is expected for significant propagation losses. Two different regimes can be identified, depending on whether propagation losses are either negligible or dominant with respect to termination losses.

1) Low propagation-loss regime, $\alpha L \ll-\ln \Gamma$ : In this case $\exp (\alpha L) \simeq 1$, while (10b) holds that $\hat{\tau} / \tau=\hat{L} / L$, hence

$$
\mathcal{A}_{p}(L, \hat{L}) \simeq \frac{2}{1+\hat{L} / L} \frac{\mathrm{e}^{-\alpha \hat{L}}}{1+\left(\eta \Delta f / B_{c}\right)^{2}},
$$

with $\mathcal{A}_{p}(L, \hat{L}) \leq 2$, reaching its upper limit for $\hat{L} \ll L$. A bias therefore exists for the projection in locating a fault at shorter distances than its actual position, unless the resonance mismatch is significant enough to offset this bias. Unfortunately, for $\hat{L} \ll L$ this mechanism becomes ineffective, since the resonance mismatch is reduced by $\eta \simeq 2 \hat{L} / L \ll 1$, thus with $\mathcal{A}_{p}>1$ even for large resonance mismatches.

This effect is visible in Fig. 2(b), where a resonance mismatch $\Delta f / B_{c}=1$, while producing a non-negligible reduction of $\mathcal{A}_{p}(L, \hat{L})$, still has a clear bias towards $\hat{L}<L$.

2) High propagation-loss regime, $\alpha L \gg-\ln \Gamma$ : When testing positions $\hat{L}$ such that $\alpha \hat{L} \gg-\ln \Gamma$, then $\hat{\tau} / \tau \simeq 1$, independently from the actual distances $L$ and $\hat{L}$, as highlighted in (12). As a result, $\eta \simeq 1$ and

$$
\mathcal{A}_{p}(L, \hat{L}) \simeq \frac{L}{\hat{L}} \frac{\mathrm{e}^{-\alpha(\hat{L}-L)}}{1+\left(\Delta f / B_{c}\right)^{2}} .
$$

The condition $\alpha L \gg-\ln \Gamma$ does not necessarily imply that $\exp (\alpha L)>1$, since $\Gamma \simeq 1$, and $-\ln \Gamma \ll 1$. Yet, in this

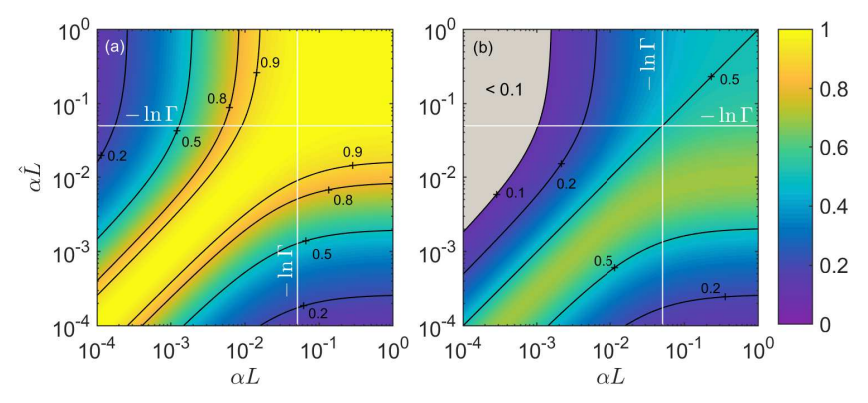

Fig. 3. Location ambiguity function $\mathcal{A}_{\rho}(L, \hat{L})$ for the correlation, for $\Gamma=\left|\Gamma_{S} \Gamma_{T}\right|=0.95$, evaluated from (25) for (a) matched resonances, i.e., $\Delta f / B_{c}=0$ and (b) $\Delta f / B_{c}=1$, for a single resonance.

case $\mathcal{A}_{p}$ is unbounded, because of the term $L / \hat{L}$ in (22). This change of behavior in $\mathcal{A}_{p}$ is clearly visible in Fig. 2, where $\mathcal{A}_{p}$ grows more rapidly for $\hat{L}<L$ than in the previous case of negligible propagation losses.

When testing positions such that $\alpha \hat{L} \ll-\ln \Gamma$ a different behavior is expected, since now $\hat{\tau} / \tau \simeq-2 \alpha \hat{L} / \ln \Gamma \ll 1$, and

$$
\mathcal{A}_{p}(L, \hat{L}) \simeq-\frac{4 \alpha L}{\ln \Gamma} \frac{\mathrm{e}^{\alpha L}}{1+\left(\eta \Delta f / B_{c}\right)^{2}} .
$$

In this case the location ambiguity function no longer depends on the position tested, but only on the actual fault position. The further the fault, the stronger the bias towards positions closer to the probe, a situation potentially made worse by the term $\exp (\alpha L)$.

\section{B. Correlation}

From (13), the ambiguity function of the correlation metric (3) is

$$
\mathcal{A}_{\rho}(L, \hat{L})=\left|\frac{\rho(L, \hat{L})}{\rho(L, L)}\right|=|\rho(L, \hat{L})|,
$$

implying that systematic errors cannot occur in this case, since $|\rho(L, \hat{L})| \leq 1$ and $\rho(L, L)=1$ by definition. Nonetheless, location ambiguity may still be observed, with $|\rho(L, \hat{L})|=1$ for $\hat{L} \neq L$.

Proceeding as in the case of the projection, applying (15)(19) to (3) yields

$$
\mathcal{A}_{\rho}(L, \hat{L})=\frac{2 \sqrt{\hat{\tau} / \tau}}{1+\hat{\tau} / \tau} \frac{1}{1+\left(\eta \Delta f / B_{c}\right)^{2}} .
$$

Both projection and correlation ambiguity functions share resonance mismatch as the main location mechanism, but correlation comes with a further mechanism, represented by the first fraction in (25), which measures the matching between the decay times $\tau$ and $\hat{\tau}$ of the actual and reference line responses. Correlation can be close to one only if both resonances and decay times are matched, as shown in Fig. 3. As for projection, correlation too presents a different behavior depending on the propagation loss regime. 
1) Low propagation-loss regime: In this case $\hat{\tau} / \tau \simeq \hat{L} / L$, thus the correlation will directly measure a mismatch between the estimated and the actual fault locations, even for perfectly matched resonances. This property is visible in Fig. 3(a), as a strip of high correlation around the fault position, with $\mathcal{A}_{\rho} \geq$ 0.95 for $\hat{L} / L \in[0.52,1.9]$. Any resonance mismatch would then significantly lower the ambiguity, as visible in Fig. 3(b), as opposed to the projection, which would still suffer from a bias towards $\hat{L} \ll L$.

2) High propagation-loss regime: For $\alpha L, \alpha \hat{L} \gg-\ln \Gamma$ correlation can no longer rely on the decay-time mismatch to locate a fault, since in this case (12) holds that $\hat{\tau} \simeq \tau$, independently from $\hat{L}$ and $L$. This phenomenon is visible in Fig. 3(a), where $\mathcal{A}_{\rho} \simeq 1$ as soon as the resonance frequencies of the actual and reference lines match, even when testing fault positions $\hat{L}$ significantly different from the actual one, L. Still, Fig. 3(b) shows that $\mathcal{A}_{\rho}<1$ as soon as resonances are mismatched.

\section{SURGE-SPECTRUM EQUALIZATION}

The analysis in Sec. IV assumed operating over a bandwidth narrow enough for the fault surge spectrum $V_{f}(\omega)$ to be regarded as constant. This is not an option in practical configurations, since $V_{f}(\omega)$, as clear from (5), is highly dispersive, introducing a disproportionate emphasis on low frequencies, eroding the contribution of high-frequency information, which may afford a better spatial resolution [24].

The standard choice in the literature is to directly measure the similarity between reference and measured transient signals [12]-[14]. It is therefore necessary to have access to a reasonably accurate model for the fault surge $v_{f}(t)$, in order to produce estimates of the transient signals $v_{m}(t ; \hat{L})$, typically by means of numerical simulations. This same information could be used more effectively in order to estimate the line resonant response $H(\omega ; L)$ starting from the measured fault transient, applying an inverse-filter equalization which, in its simplest form, reads

$$
\hat{H}(\omega ; L)=V_{m}(\omega) / V_{f}(\omega) .
$$

It is thus possible to measure the similarity between transfer functions by computing their projection

$$
\mathcal{P}(L, \hat{L})=[\hat{H}(\omega ; L) \mid H(\omega ; \hat{L})]
$$

and the respective correlation location metric. As proven in Sec. VI, equalization is not beneficial because it gives access to an assumed better accuracy at higher frequencies, but rather because it reduces the influence of low frequencies where the line may not yet resonate, a configuration which would result in a very poor location accuracy (cf. Sec. VI).

It is worth noticing that it has recently been shown in [16] that a precise knowledge of the surge spectrum $V_{f}(\omega)$ is not necessary, and that a partial equalization is sufficient in order to significantly improve location accuracy.

\section{Single-PhASE OVERHEAD LINE}

The predictions and conclusions drawn in Sec. IV are first validated for the case of a single-phase overhead line, above

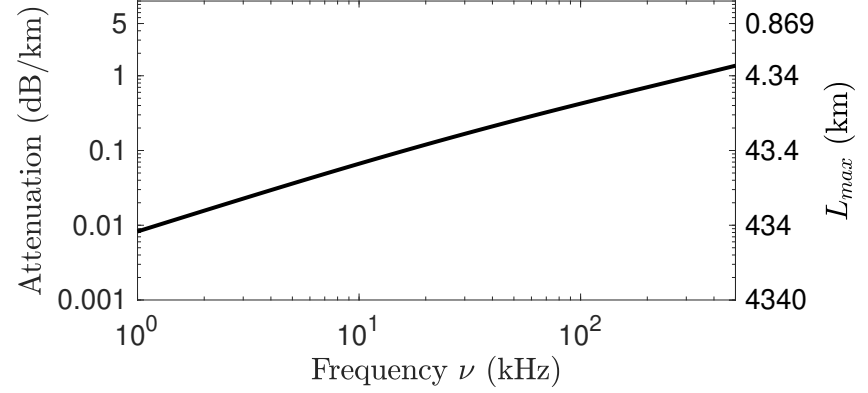

Fig. 4. Propagation losses for the overhead line studied in Section VI. The second vertical axis on the right shows the maximum fault distance $L_{\max }$ required for the line to potentially resonate.

a lossy ground. A low-impedance shunt fault is found at a distance $L$, approximatively acting as a termination as in Fig. 1, a configuration for which (8) provides an accurate model of the transient recorded by a probe. The line consists of an aluminium conductor of radius $15 \mathrm{~mm}, 14.1 \mathrm{~m}$ above a homogeneous soil with relative electric permittivity $\epsilon_{g}=10$ and conductivity $\sigma_{g}=10 \mathrm{mS} / \mathrm{m}$.

Propagation losses are expected to be highly frequency dependent, and thus require an accurate modeling of the line per-unit-length parameters. In this respect, we have opted to use the model presented in [28], which improves approximate models such as Carson's. This choice is fundamental in order to accurately reproduce the rapidly increasing propagation losses expected for the ground mode, as opposed to the choice of fixed-parameter models, likely leading to underestimating the impact of propagation losses. The model presented in [28] will also be applied in Sec. VII to a three-phase overhead line.

The line's left end, where the transient voltage is measured, is terminated by an impedance $Z_{T}=10 \mathrm{k} \Omega$, representing the high input impedance of a power transformer at frequencies above $1 \mathrm{kHz}$ [1], and a shunt fault of impedance $Z_{S}=10 \Omega$ is found at the right end, corresponding respectively to reflection coefficients $\left|\Gamma_{T}\right| \simeq 0.89$ and $\left|\Gamma_{S}\right| \simeq 0.97$, below $500 \mathrm{kHz}$; the termination loss factor is $-\ln \Gamma=-\ln \left|\Gamma_{T} \Gamma_{S}\right| \simeq 0.15$. The line attenuation is shown in Fig. 4, together with the maximum distance $L_{\max }$ necessary for the line to support resonances, approximatively identified by the condition $2 \alpha L_{\max }=1$. These results indicate that fault surge information above 500 $\mathrm{kHz}$ would likely be inaccessible apart in case of faults less than $4 \mathrm{~km}$ away from the probe.

Three fault distances were considered, namely $L=0.5,2$ and $16 \mathrm{~km}$, for a fault surge described by (5), with a rise time $T_{r}=50 \mu \mathrm{s}$. Projection and correlation metrics were then computed for 5000 test positions $\hat{L}$, ranging from 0.1 to 20 $\mathrm{km}$, for both transient spectra and the line transfer functions, as discussed in Sec. V. The main goal being to compare the location accuracy of both metrics, depending on propagation losses and the bandwidth of the recorded transient data.

Fig. 5 shows the resonant response of the line, as defined in (7), for the $1-500 \mathrm{kHz}$ frequency range, as a function of the fault distance $L$. The first line resonance is found to be about four times stronger than the second one, thus playing a dominant role in the fault spectra and the location metrics. Higher- 


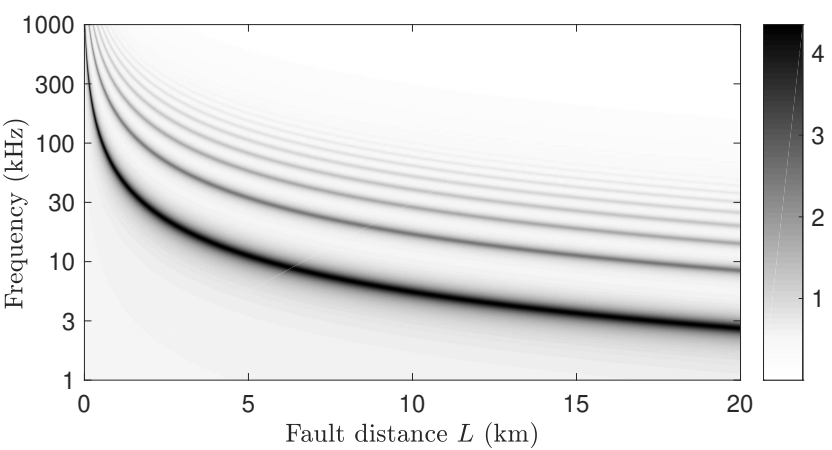

Fig. 5. Resonant response of the overhead line, as a function of fault distance.

order resonances appear to become increasingly damped by propagation losses, and could hardly contribute to improve the location accuracy.

Knowing propagation and termination losses, the spatial resolution enabled by a single resonance can be predicted from (11). Fig. 6 shows that for faults more than $1 \mathrm{~km}$ away from the probe the spatial resolution would not improve by extending the tested frequency bandwidth beyond about $50 \mathrm{kHz}$, due to increasingly high propagation losses. The resonant-line limit, corresponding to the condition $2 \alpha L-\ln \Gamma=1$, approximatively identifies the maximum frequency below which the spatial resolution is expected to improve by accessing a larger bandwidth during the transient recording, i.e., by increasing the sampling rate. This boundary covers the first three resonances of the line in Fig. 5. The results in Sec. VI-A support these theoretical predictions.

\section{A. Line transfer functions}

Results for the similarity between transfer functions are discussed first, since in this case there is no emphasis from the surge spectrum on low frequencies, leading to a simpler interpretation of the results.

Transients are first supposed to be recorded over the bandwidth from 1 to $50 \mathrm{kHz}$. Fig. 7(a) shows that a fault at $0.5 \mathrm{~km}$ is poorly located, since in this case none of the line resonances

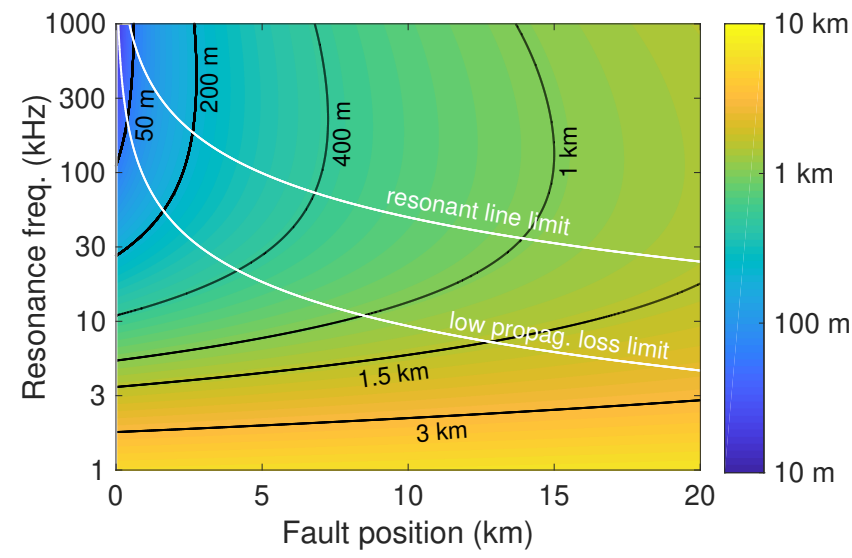

Fig. 6. Single-resonance spatial resolution predicted by (11) for the overhead line, with the limits for a resonant line $(2 \alpha L-\ln \Gamma=1)$ and negligible propagation loss regime $(2 \alpha L=-\ln \Gamma)$ shown. These results assume a resonance match and should thus be regarded as a best-case prediction. is accessible, as confirmed by Fig. 5. The projection presents a peak around $1.2 \mathrm{~km}$, where the first resonance first appears in the $1-50 \mathrm{kHz}$ bandwidth, while it takes a significantly lower value over the fault position, where only the lower end of the resonance tail is accessible. No such bias is observed for the correlation, thanks to the local normalization taking into account the actual norm of the transfer functions. The strongly reduced spatial resolution of the correlation is also explained by the fact that only decay-time matching is available in this case, as discussed in Sec. III.

Both metrics correctly located a fault at $2 \mathrm{~km}$, having now access to the first line resonance. For a fault at $16 \mathrm{~km}$ the spatial resolution is significantly reduced, in accordance with the results estimated in Fig. 6 .

A secondary peak is observed in Fig. 7(c) when simulating a tested fault distance $\hat{L}=16 / 3 \simeq 5.3 \mathrm{~km}$, since in this case the actual and tested lines have their respectively second and first resonances matched at $10.4 \mathrm{kHz}$, as visible in Fig. 5. As explained in Section IV-A, the normalized projection should be expected in this case to present a higher peak for $\hat{L}<L$. In fact, the peak at $5.3 \mathrm{~km}$ is smaller than the one at 16 $\mathrm{km}$ because only one of the two line resonances is matched, thus reducing the overall projection. A similar phenomenon is found for the fault at $2 \mathrm{~km}$, with a secondary peak at $2 \times 3=$ $6 \mathrm{~km}$. While these two secondary peaks occur for the same reason, their amplitudes is unequal: the peak at $5.3 \mathrm{~km}$ for a fault at $16 \mathrm{~km}$ is significantly larger, almost reaching the same value as at the actual fault position. This bias was indeed predicted in Sec. IV-A for the projection for $\hat{L}<L$, while the correlation does not present any such bias.

The results obtained applying the same analysis to data covering the 1-500 kHz range are shown in Fig. 8. Several notable differences are observed. At $0.5 \mathrm{~km}$ the fault is now located with high accuracy, since two line resonances are now accessible. The spatial resolution, close to $\pm 50 \mathrm{~m}$, agrees with Fig. 6, while a secondary peak is observed for the projection at about $180 \mathrm{~m}$, due to two resonances matching around $350 \mathrm{kHz}$.

The intensity of the secondary peaks in the projection increases for faults at larger distances, creating the potential for ambiguity due to multiple viable locations where at least two resonances match, as in Fig. 8(b). For a fault at $16 \mathrm{~km}$, the projection presents several peaks less than $3 \mathrm{~km}$ from the probe, caused by several resonance matches, as visible in Fig. 5. These results confirm that the risk of a systematic error increases with the fault distance, as soon as propagation losses increase.

Furthermore, although the bandwidth increases tenfold, the spatial resolution does not improve significantly for a fault at $2 \mathrm{~km}$, while at $16 \mathrm{~km}$ it rather worsens, consistently with the results in Fig. 6. The main effect of an increased bandwidth is the appearance of secondary peaks closer to the probe, due to new accessible resonances for shorter tested distances, as clear from Fig. 5.

The correlation is not affected by any of these phenomena, with systematically lower secondary peaks. Nonetheless, it presents ambiguity as soon as the conditions for a high propagation-loss regime are met (cf. Sec. IV-B). Fig. 9(a) shows results for the correlation for a fault at $8 \mathrm{~km}$, when 

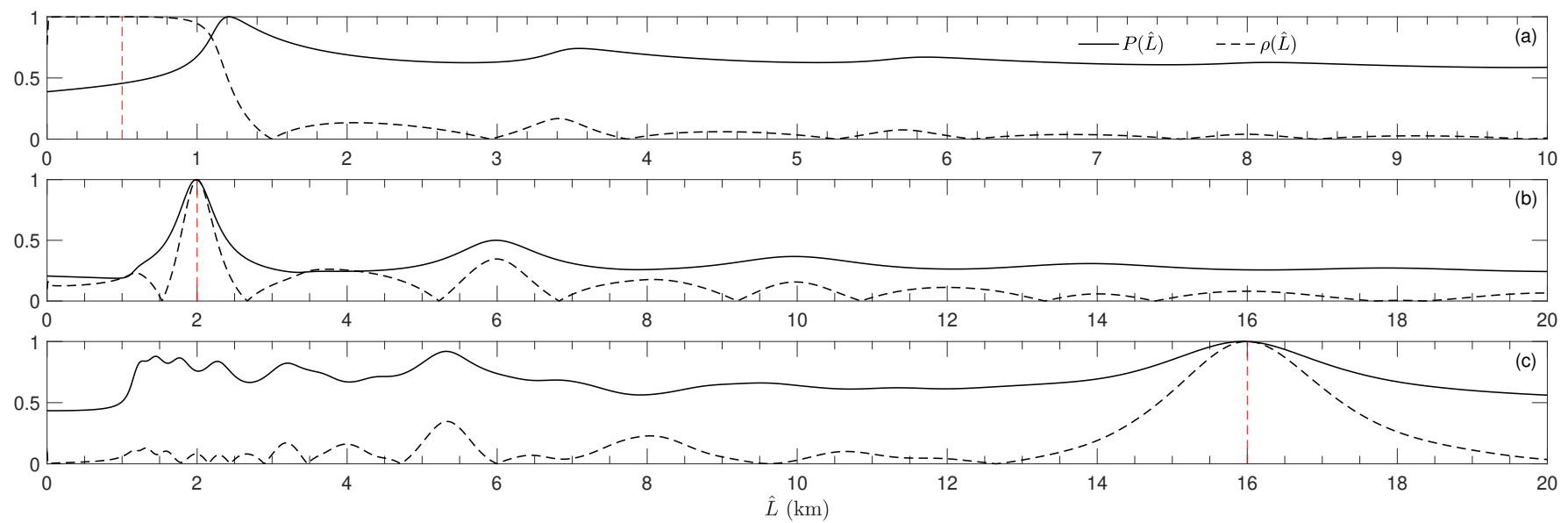

Fig. 7. Location results of projection and correlation metrics for three faults at a distance of (a) $0.5 \mathrm{~km}$, (b) $2 \mathrm{~km}$ and (c) $16 \mathrm{~km}$ along an overhead line, using line transfer functions over a $1-50 \mathrm{kHz}$ bandwidth.
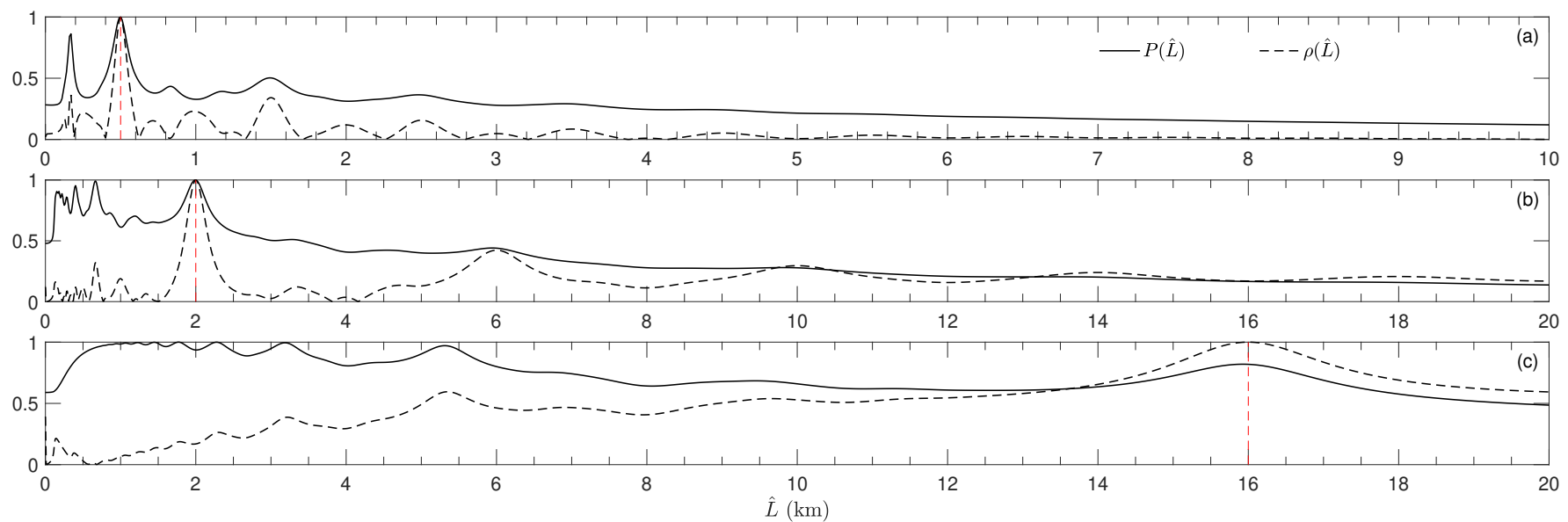

Fig. 8. Location results of projection and correlation metrics for three faults at a distance of (a) $0.5 \mathrm{~km}$, (b) $2 \mathrm{~km}$ and (c) $16 \mathrm{~km}$ along an overhead line, using line transfer functions over a $1-500 \mathrm{kHz}$ bandwidth.
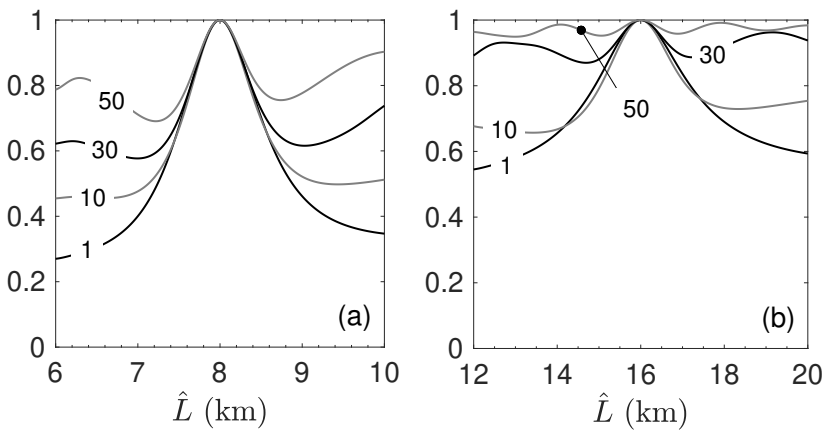

Fig. 9. Location results for a fault at (a) $8 \mathrm{~km}$ and (b) $16 \mathrm{~km}$, for correlation applied to line transfer functions data below $500 \mathrm{kHz}$, restricted to frequencies higher than 1,10,30 and $50 \mathrm{kHz}$, where high propagation-loss conditions are expected, highlighting increasing location ambiguity.

access to low frequency data is limited, thus relying on higher-order resonances subject to higher propagation losses: the correlation decays less away from the fault. For a more distant fault at $16 \mathrm{~km}$, higher propagation losses are expected: Fig. 9(b) shows clear signs of ambiguity even for the correlation. It is therefore important to minimize the tested bandwidth, avoiding contributions from higher frequencies, where propagation losses exceed those in the line terminations. Fig. 6 shows the maximum frequency below which negligible propagation losses can be expected. Attempting to locate a fault at higher frequencies, ambiguity must be expected: at 8 $\mathrm{km}$, this degradation occurs above $10 \mathrm{kHz}$.

\section{B. Transient signals}

When directly applying projection and correlation metrics to transient signals, as done in the literature, the surge spectrum $V_{f}(\omega)$ is expected to highly emphasize the lower end of the frequency range. Results obtained for data covering the 1$500 \mathrm{kHz}$ bandwidth in Fig. 10 present a dramatic loss of accuracy. Clearly, the reason for these results is to be found in the dominant contribution of the $1 \mathrm{kHz}$ region, where Fig. 5 shows that no resonance is accessible for faults less than $10 \mathrm{~km}$ from the probe, explaining the poor accuracy in Fig. 10. Even under these degraded conditions, the correlation reaches its peak value at the correct fault positions, thanks to its additional location mechanism based on the decay time $\tau$. 

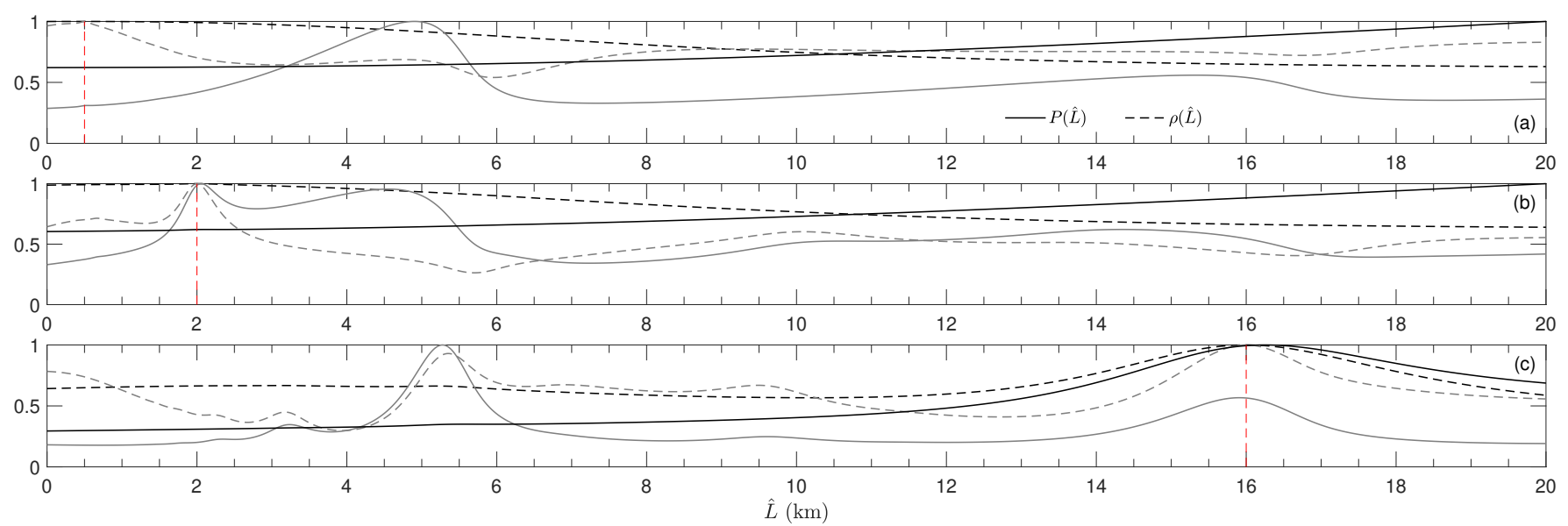

Fig. 10. Location results of projection and correlation metrics for three faults at a distance of (a) $0.5 \mathrm{~km}$, (b) $2 \mathrm{~km}$ and (c) $16 \mathrm{~km}$ along an overhead line, using full-transient spectra sampled over a 1-500 kHz bandwidth (black lines) and 10-500 kHz bandwidth (gray lines).

It could be expected that increasing the minimum frequency from 1 to $10 \mathrm{kHz}$ should partially reduce this issue and thus improve the location accuracy. The effects of this change are shown in Fig. 10 as grey lines: while the correlation benefits from it with an improved spatial resolution, the projection displays peaks at erroneous positions for a fault at 0.5 and $8 \mathrm{~km}$. In both cases, starting at either 1 or $10 \mathrm{kHz}$, the spatial resolution is significantly worse than when using transfer functions over a narrower bandwidth, between 1 and $50 \mathrm{kHz}$ (cf. Fig. 7). This comparison demonstrates the benefits of using a priori information about the surge signal to estimate the line transfer functions, as argued in Sec. V.

Surge equalization is therefore not intended to give access to high frequency information which, as proven in the previous section, would not necessarily provide a better accuracy, but rather to avoid that contributions at lower frequencies out of resonance drown out contributions from higher-frequency resonances that would otherwise provide an accurate fault location.

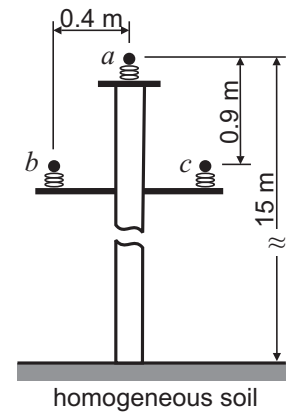

(a)

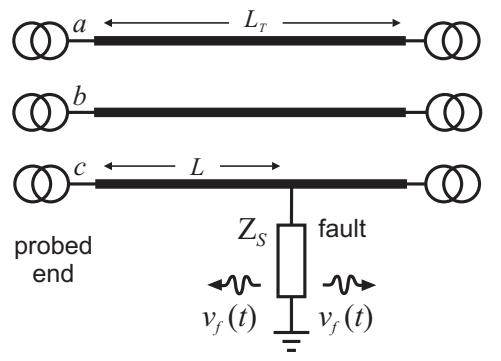

(b)
Fig. 11. Three-phase line configuration: (a) cross section (b) single-phase to ground fault. The ends of each phase are terminated by a load with an impedance $Z_{T}=10 \mathrm{k} \Omega$, while the fault has an impedance $Z_{S}=10 \Omega$.

\section{THREE-PHASE OVERHEAD LINE}

The analysis for a single-phase line has clarified the physical mechanisms behind the location accuracy of TBM and the risk of location errors in lossy lines. In practice, the vast majority of power-transmission lines are based on threephase configurations. Fig. 11(a) shows the cross-section of the transmission line studied in this section, a $10 \mathrm{kV}$ overhead power distribution line commonly employed in China. The single-phase configuration studied in Sec. VI was chosen to correspond to one of the lower conductors of this three-phase line, in order to allow a direct comparison.

This section sets out to verify that the phenomena identified in the case of a single-phase line also apply to three-phase lines. To this end, some implications of the modal propagation theory are discussed in Sec. VII-A, covering the role of ground and aerial modes, while Sec. VII-B explains how shunt faults alter the propagation along a three-phase line in a significantly different way with respect to a single-phase line. Sec. VII-C presents results confirming that the risk of location ambiguity is still governed by the same phenomena identified in Sec. IV and presents strong similarities with the case of a single-phase line. Sec.VII-D focuses on the spatial resolution of correlationbased fault location, explaining why it can significantly change depending on the fault distance and the tested bandwidth, thanks to insights brought by modal theory.

While shunt faults can take a number of different configurations, appearing across phases as well as toward the ground [2], this section will only focus on the case of a single-phaseto-ground fault, occurring on phase $c$, as in Fig. 11(b). This configuration is the most likely shunt fault found in practice [2, Sec. 3]. Clearly, the procedure used for this analysis can also be applied to other kind of faults, e.g., phase-to-phase faults: a thorough comparison of how each fault affords a different location accuracy is out of the scope of the present paper, which is instead focused on the impact of a line's losses.

The line under consideration has a length $L_{T}=50 \mathrm{~km}$ and runs above a flat homogeneous ground, with relative electric permittivity $\epsilon_{g}=10$ and conductivity $\sigma_{g}=10 \mathrm{mS} / \mathrm{m}$. The line conductors have a radius equal to $15 \mathrm{~mm}$ and are assumed to be made of aluminium. The three-phase line is terminated at both ends by power transformers, modelled by termination impedances $Z_{T}=10 \mathrm{k} \Omega$ [1] applied to each conductor, while 


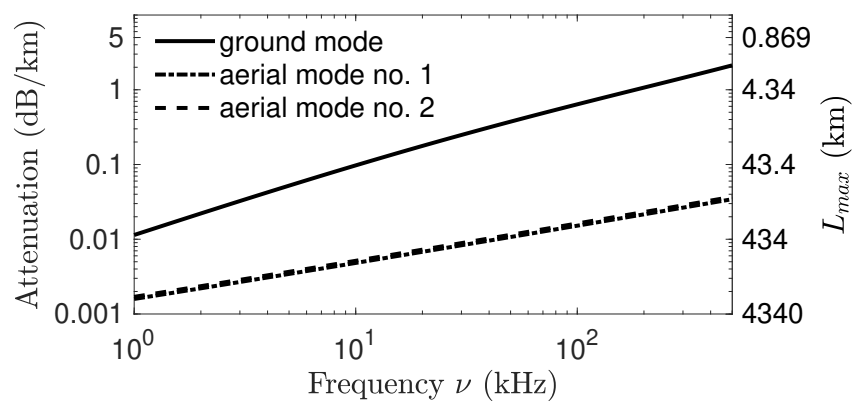

Fig. 12. Propagation losses for the three modes supported by the three-phase line described in Fig. 11(a).

the shunt fault has an impedance $Z_{S}=10 \Omega$.

\section{A. Modal propagation theory}

Signal propagation along a multi-conductor line is better understood through a modal description, where signals are represented as a linear combination of voltage and current patterns, or modes [27]. The modal representation can be derived by solving the following eigenvalue problem

$$
(\mathbf{Y}(\omega) \mathbf{Z}(\omega)) \mathbf{T}(\omega)=\mathbf{T}(\omega) \gamma^{2}(\omega)
$$

where $\mathbf{Y}(\omega)$ and $\mathbf{Z}(\omega)$ are the per-unit-length admittance and impedance matrices of the line. The columns of matrix $\mathbf{T}(\omega)$ correspond to the eigenvectors of the matrix $\mathbf{Y}(\omega) \mathbf{Z}(\omega)$, while their eigenvalues are found in the diagonal matrix $\gamma^{2}(\omega)$. The per-unit-length matrices were computed by means of the model described in [28], also applied to the single-phase line discussed in Sec. VI.

The voltages of each overhead conductor at a distance $x$, $V_{a}(x)$ through $V_{c}(x)$, can be arranged in the voltage vector $\mathbf{V}(x)=\left[V_{a}(x), V_{b}(x), V_{c}(x)\right]^{\mathrm{T}}$; a current vector $\mathbf{I}(x)$ can be defined in a similar way. Modal propagation theory states that the evolution of voltage and current vectors along a uniform line can be expressed in terms of modal current vectors $\mathbf{I}_{m 0}^{ \pm}$

$$
\begin{aligned}
\mathbf{V}(x) & =\mathbf{Z}_{c} \mathbf{T}\left[\mathbf{P}^{+}(x) \mathbf{I}_{m 0}^{+}+\mathbf{P}^{-}(x) \mathbf{I}_{m 0}^{-}\right] \\
\mathbf{I}(x) & =\mathbf{T}\left[\mathbf{P}^{+}(x) \mathbf{I}_{m 0}^{+}-\mathbf{P}^{-}(x) \mathbf{I}_{m 0}^{-}\right]
\end{aligned}
$$

where $\mathbf{P}^{ \pm}(x)=\exp (\mp \gamma x)$ are the forward $(+)$ and backward (-) modal propagators. The characteristic impedance matrix is defined as $\mathbf{Z}_{c}=\mathbf{Z} \mathbf{T} \boldsymbol{\gamma}^{-1} \mathbf{T}^{-1}$. The diagonal matrix $\gamma$ contains the complex propagation constants of each mode and is defined as the principal square root of the eigenvalue matrix $\gamma^{2}$, to ensure passivity. In the following discussions, the first eigenvalue and eigenvector will correspond to the ground mode, and the remaining two to aerial modes.

Fig. 12 shows the propagation attenuation for these modes for the line in Fig. 11, up to $500 \mathrm{kHz}$. The attenuation of each mode is obtained by computing the modulus of the diagonal terms in the forward propagator $\mathbf{P}^{+}(x)$, i.e., $\exp \left(-\operatorname{Re} \gamma_{i} x\right), i \in[1,3]$. The distance $L_{\max }$ on the righthand scale indicates the maximum distance beyond which propagation losses would be sufficient to make the line nonresonant, as described in Sec. VI. Ground-mode losses are very similar to those displayed in Fig. 4 for a single-phase line with ground return, but slightly higher above $100 \mathrm{kHz}$.

Results shown in the rest of this section were computed by means of an in-house transmission-line code based on modal theory. On top of the possibility to include accurate highfrequency ground loss models as [28], it provides direct access to modal quantities, upon which our analysis is based.

\section{B. Interaction and dissipation from terminations and faults}

The marked difference in ground- and aerial-mode propagation losses should be expected to lead to a significantly different behavior in the location ambiguity of correlationand projection-based metrics. As proven in Sec. IV, location ambiguity becomes likelier as soon as propagation losses exceed those in the line terminations and the fault. Besides, as propagation losses increase, (11) predicts that the spatial resolution afforded by a line resonance should be expected to worsen, as the length of the resonant line increases.

Unfortunately, it is not possible to directly transpose the theory presented in Sec. III for a single-phase line to the case of a three-phase line. Line resonances now involve a complex combination of modal patterns, which travel along the line at different speeds and rates of attenuation. Furthermore, discontinuities such as terminations and faults do not act independently on each line mode, and may further couple ground and aerial mode contributions, as proven in this section. The existence of this modal coupling implies that it would be incorrect to expect that this complexity could be simplified by filtering out the ground mode, as often done in transient monitoring.

Transient signals propagating toward any discontinuity are only partially reflected back. Expressing the impinging signals through their modal-current vector $\mathbf{I}_{m}^{+}$, the reflected modalcurrent vector $\mathbf{I}_{m}^{-}$can be expressed in terms of the modal reflection matrix $\boldsymbol{\Gamma}_{D}$, such that $\mathbf{I}_{m}^{-}=\boldsymbol{\Gamma}_{D} \mathbf{I}_{m}^{+}$, where $\boldsymbol{\Gamma}_{D}=$ $\mathbf{T}^{-1}\left(\mathbf{Z}_{D}+\mathbf{Z}_{c}\right)^{-1}\left(\mathbf{Z}_{D}-\mathbf{Z}_{c}\right) \mathbf{T} ; \mathbf{Z}_{D}$ is the impedance matrix of the discontinuity.

For the line in Fig. 11, the modal reflection matrix, evaluated at $10 \mathrm{kHz}$, is

$$
\boldsymbol{\Gamma}_{T} \simeq\left(\begin{array}{ccc}
0.82 & 0 & 0 \\
0 & 0.95 & 0 \\
0 & 0 & 0.95
\end{array}\right)
$$

and thus acts on each mode separately, i.e., similarly to the case of the single-phase line seen in Sec. III. The ground mode is subject to significantly higher termination losses than aerial modes.

Conversely, the reflection matrix $\Gamma_{S}$ for the $10 \Omega$ fault in Fig. 11 is

$$
\boldsymbol{\Gamma}_{S} \simeq\left(\begin{array}{ccc}
-0.64 & 0.12 & -0.19 \\
0.44 & -0.080 & 0.13 \\
-0.78 & 0.14 & -0.23
\end{array}\right) .
$$

The fact that $\boldsymbol{\Gamma}_{S}$ is a full matrix means that the fault couples the three modes together. In particular, the first column of $\boldsymbol{\Gamma}_{S}$ measures how a ground mode impinging onto the fault is reflected back with roughly similar intensity across all three 
modes, whereas the two other columns, concerning aerialmode reflection, have significantly smaller coefficients. Hence, upon the very first interaction with the fault, the ground mode would partially transfer energy to the aerial modes.

A better understanding of how this fault affects the modal mix is gained by looking into the eigendecomposition of $\boldsymbol{\Gamma}_{S}$, which yields its eigenvectors $\left\{\mathbf{w}_{i}\right\}, i \in[1,3]$

$$
\left(\mathbf{w}_{1}\left|\mathbf{w}_{2}\right| \mathbf{w}_{3}\right) \simeq\left(\begin{array}{ccc}
0.58 & -0.32 & -0.24 \\
-0.39 & -0.29 & 0.24 \\
0.70 & -0.90 & 0.94
\end{array}\right)
$$

and eigenvalues $\left\{\lambda_{i}\right\}=\left\{\Gamma_{o}, 0,0\right\}$, with $\Gamma_{o}=-0.95$, establishing that $\boldsymbol{\Gamma}_{S}$ has rank one. Hence, representing the impinging modal currents as a linear combination of the eigenvectors $\left\{\mathbf{w}_{i}\right\}$

$$
\mathbf{I}_{m}^{+}=\sum_{i=1}^{3} \alpha_{i} \mathbf{w}_{i}
$$

the modal currents reflected by the fault would be given by $\mathbf{I}_{m}^{-}=\Gamma_{o} \alpha_{1} \mathbf{w}_{1}$, hence the fault would respond by reflecting a fixed modal current mix, proportional to $\mathbf{w}_{1}$, independently of the one impinging on the fault. In case the line terminations reflected back this pattern unaltered towards the fault, this same pattern would undergo a reflectivity equal to -0.95 , i.e., very close to that observed for the single-phase line discussed in Sec. VI. Conversely, impinging currents described by any linear combination of $\mathbf{w}_{2}$ and $\mathbf{w}_{3}$ would result in no reflection from the fault, travelling unaffected through it. This strong difference in the behavior of the fault explains the existence of two groups of resonances: a) section resonances, where a significant fraction of energy is trapped in one of the sections in which the line is divided by the fault, where the fault presents a strong reflectivity and b) whole-line resonances, where the fault presents a much weaker interaction with the transient. In both cases, it is no longer possible to assume a negligible transmission across the fault, as done for a singlephase line. By the same token, it is therefore clear that it would be wrong to interpret fault reflectivities smaller than one in $\boldsymbol{\Gamma}_{S}$ as a signature of power loss, as done in Sec. III.

It is therefore incorrect to expect a unique value for the loss factor contributed by the fault, as it now depends on the modal mix, which furthermore changes over time as the ground mode decays more rapidly than aerial modes. Nonetheless, it is possible to derive bounds for the fraction of power dissipated within the fault, as explained in Appendix A. For a transient impinging onto the fault, the fraction of dissipated power is between 0 and $8 \%$, indicating that at least $92 \%$ of the power is partially reflected and transmitted through the fault and will therefore further interact with it, thus actively contributing to the line resonances. This figure suggests an effective reflectivity larger than $\sqrt{0.92} \simeq 0.96$, thus similar to the single-phase reflectivity.

Fig. 13 shows ground- and aerial-mode contributions to the transfer function between the fault and a probe at the left end of phase $c$. It can be noticed how the most strongly excited resonances still present a sensitivity to the fault position similar to that in Fig. 5 for a single-phase configuration, with

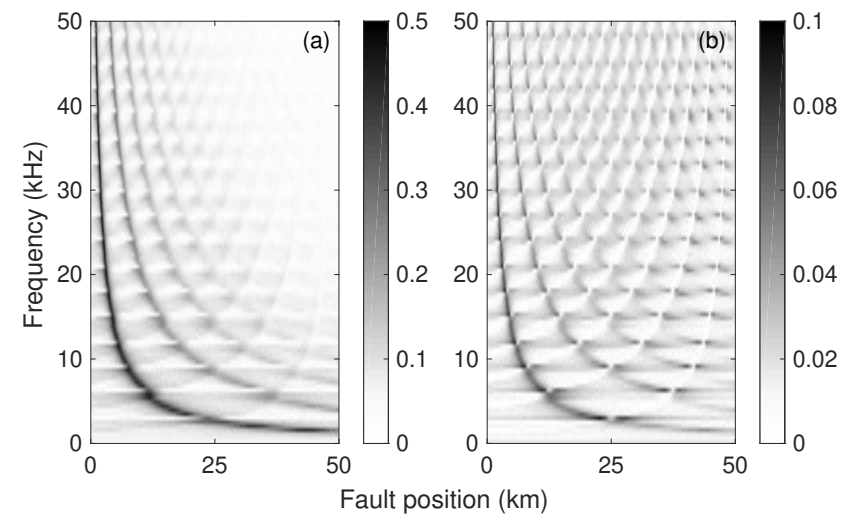

Fig. 13. Modulus of the transfer functions between a fault and a probe on phase $c$, showing contributions from (a) ground and (b) aerial modes.

frequencies of resonance inversely proportional to the fault distance. Such dependence requires that part of the transient be trapped within the left section of the line, subject to strong reflection from the fault, or section resonances. But another set of resonances now appears at much lower frequencies, which could only be sustained if the full length of the line were involved. Moreover, these resonances appear to be more strongly supported by aerial modes, coupling only partially to the ground mode. As a result, they do not present any significant difference in their average intensity even for faults at $50 \mathrm{~km}$, as opposed to the ground-mode contribution, which rapidly decreases with the fault distance (cf. Fig. 12).

Fig. 14 shows these same transfer functions in detail for three fault distances. For a fault at $2 \mathrm{~km}$ from the probe, two sets of interlaced resonances are visible in Fig. 14(a): a set with a periodicity of about $74 \mathrm{kHz}$, corresponding to a resonant line about $2 \mathrm{~km}$ long (section resonances), and another with a periodicity of about $3 \mathrm{kHz}$, consistent with a resonant line 50 $\mathrm{km}$ long (whole-line resonances). A very similar behavior is observed for ground- and aerial-mode contributions, explained by the eigendecomposition (32). For a fault at $16 \mathrm{~km}$, the ground-mode contribution becomes relegated at frequencies below $200 \mathrm{kHz}$, where section resonances can be still distinguished, with a reduction by a factor eight in their periodicity, proportional to the change in the fault position, passing from 2 $\mathrm{km}$ to $16 \mathrm{~km}$. At higher frequency, only whole-line resonances are observed. This trend becomes clearer for a fault at $48 \mathrm{~km}$, where the aerial modes are dominant above $75 \mathrm{kHz}$. It can be noticed how whole-line resonances approximatively maintain the same periodicity independently from the fault position. This does not mean that it does not affect them: the transfer functions in Fig. 14 present significantly different patterns that TBM can exploit to locate a fault, as proven in Sec. VII-C.

\section{Fault location and ambiguity}

Only fault metrics between transfer functions are discussed, since the case of transients was already proven in Sec. VI to be less effective and potentially inaccurate. The numerical analysis mirrors that presented in Sec. VI-A for a single-phase line, for the sake of a simpler comparison. Only results for data 


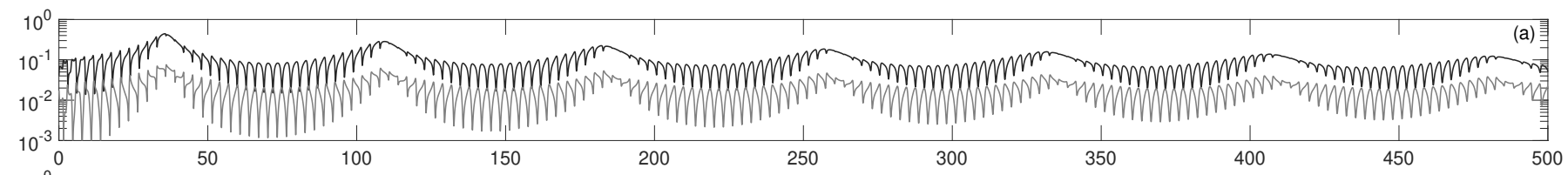

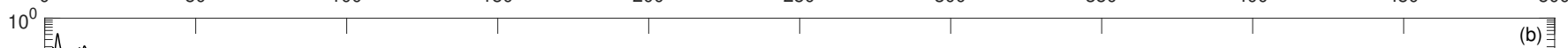

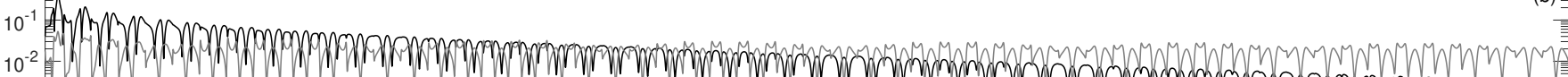

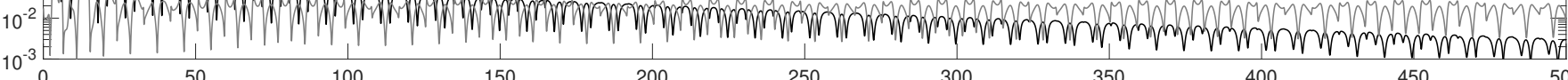

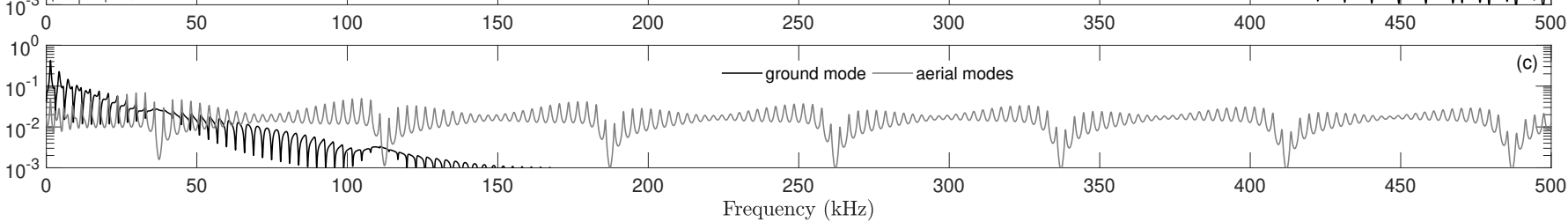

Fig. 14. Transfer function between a fault and a probe at the left end of phase $c$, showing contributions from ground and aerial modes, for a fault at a distance of: (a) $2 \mathrm{~km}$, (b) $16 \mathrm{~km}$ and (c) $48 \mathrm{~km}$.

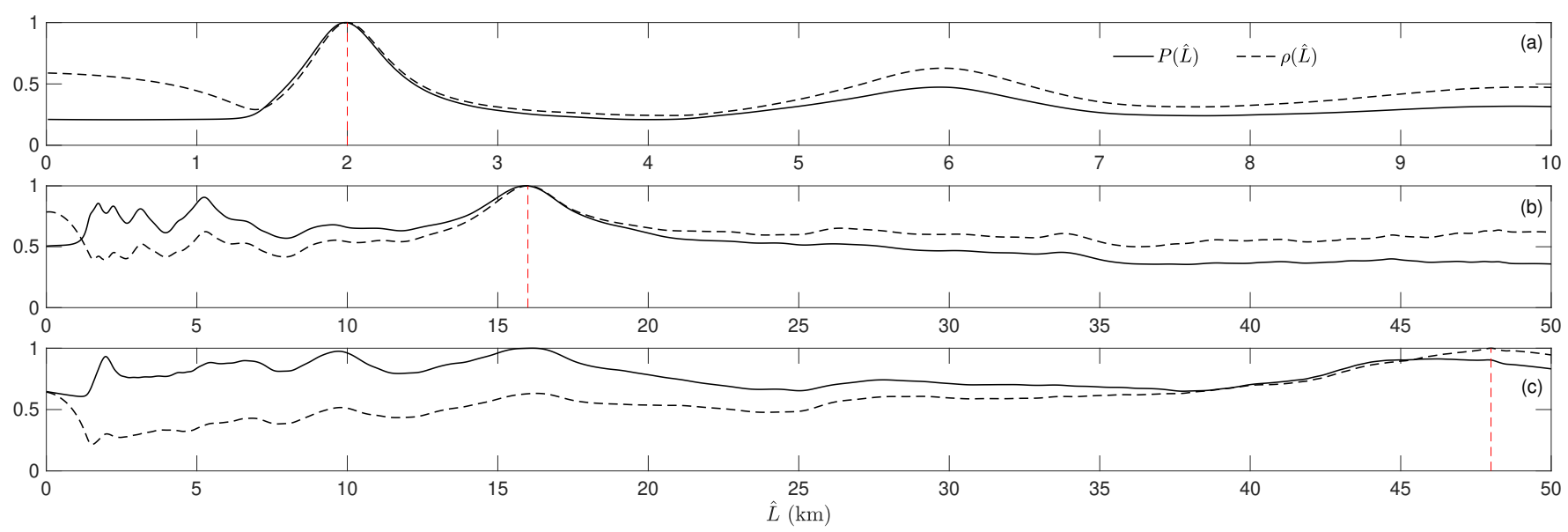

Fig. 15. Location results of projection and correlation metrics for three faults at a distance of (a) $2 \mathrm{~km}$, (b) $16 \mathrm{~km}$ and (c) $48 \mathrm{~km}$ along the three-phase overhead line in Fig. 11, using line transfer functions over a 1-50 kHz bandwidth.
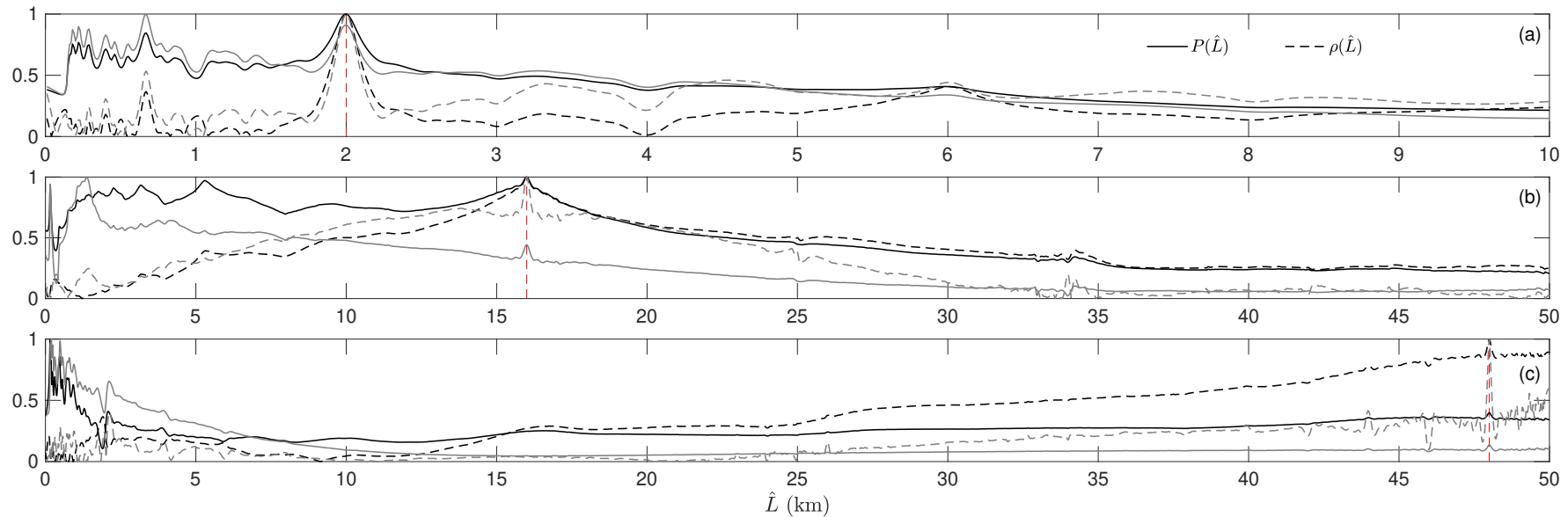

Fig. 16. Location results of projection and correlation metrics for three faults at a distance of (a) $2 \mathrm{~km}$, (b) $16 \mathrm{~km}$ and (c) $48 \mathrm{~km}$ along the three-phase overhead line in Fig. 11, using line transfer functions over a 1-500 kHz (black lines) and 50-500 kHz (grey lines) bandwidth. 
collected at the phase $c$ are shown, since no major difference was observed with the other phases.

Fig. 15 presents the correlation metric for faults at 2, 16 and $48 \mathrm{~km}$ from the probed end, using data limited to $50 \mathrm{kHz}$. Overall, the correlation presents no ambiguity, while the projection has spurious peaks increasing in intensity as the fault gets further from the probe. These results compare well with those shown in Fig. 7 for a single-phase line. This agreement is explained by the fact that the ground mode dominates the transfer functions below $50 \mathrm{kHz}$ (cf. Fig. 14). For a fault at $48 \mathrm{~km}$, a clear location bias is observed for the projection, which reaches its maximum at $16 \mathrm{~km}$, explained by matching resonance frequencies and the increasing propagation losses at this distance, as predicted in Sec. IV-A.

Similar conclusions are reached when comparing results for a fault at $2 \mathrm{~km}$ in Fig. 16(a) and Fig. 8(b), based on data extending to $500 \mathrm{kHz}$. In fact, in this case the ground mode still dominates the transfer function between the fault and the probe. But Fig. 14(b) shows that at $16 \mathrm{~km}$ the ground mode is expected to be mostly contributing below $200 \mathrm{kHz}$, with high-frequency data now dominated by aerial modes. This difference shows up in Fig. 16(b), where the location ambiguity now subdues with respect to the case of a single-phase line. The spatial resolution also presents a clear improvement, thanks to the lower propagation losses of the aerial modes. For a fault at $48 \mathrm{~km}$ a very strong bias occurs for the projection, with a peak observed at $160 \mathrm{~m}$, due to highfrequency resonances overlapping with those of a candidate fault tested at $160 \mathrm{~m}$ from the probe.

The beneficial role of whole-line resonances becomes apparent when filtering out low-frequency data where the ground mode dominates. Results in grey color in Fig. 16, computed from data covering $50-500 \mathrm{kHz}$, prove that the correlation performance dramatically improves for faults at 16 and 48 $\mathrm{km}$ distance, where the ground-mode contribution reaching the probe at the left-end termination is negligible. On the other hand, projection ambiguity worsens. It is worth noting that the spatial resolution for a fault at $48 \mathrm{~km}$ is in this case similar to that of a fault at $2 \mathrm{~km}$ from the probe, where the ground mode dominates.

\section{Bandwidth and spatial resolution of the correlation metric}

The correlation in Figs. 15 and 16 displays very different spatial resolutions depending on the fault distance and tested bandwidth. These results can be understood only through the lens of modal theory. In light of the analysis presented in Sec. VII-B, for faults close to the probe, the ground mode is expected to dominate, as confirmed by Fig. 14(a), similarly to a single-phase line. Hence, as shown in Fig. 6 for a singlephase line, increasing the tested bandwidth would not improve the spatial resolution indefinitely, as propagation losses rapidly increase with the frequency. Fig. 17(a) supports this interpretation, with the spatial resolution steadily improving up to 200 $\mathrm{kHz}$, but presenting only a minor improvement when extended to $500 \mathrm{kHz}$, consistently with the results in Fig. 6 .

For further faults this situation is reversed, with highfrequency contributions, mostly due to aerial modes, now
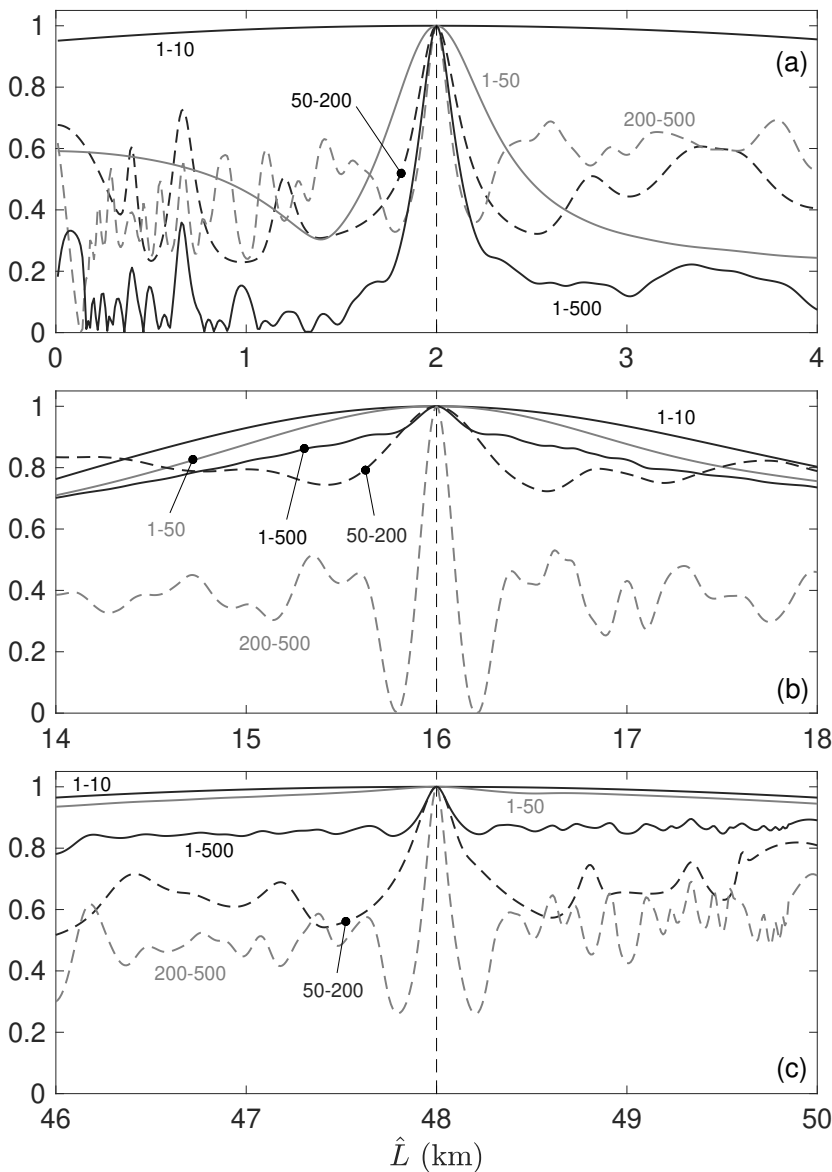

Fig. 17. Spatial resolution of the transfer-function correlation for the threephase line in Fig. 11, depending on the tested bandwidth (expressed in $\mathrm{kHz}$ for each curve) and a fault at (a) $2 \mathrm{~km}$, (b) $16 \mathrm{~km}$ and (c) $48 \mathrm{~km}$ distance.

displaying negligible propagation losses even at $500 \mathrm{kHz}$. Fig. 17(b) shows how, for a fault at $16 \mathrm{~km}$, the spatial resolution is more significantly improved for high-frequency data, e.g., covering the 200-500 bandwidth, than when including lowfrequency contributions which, at such large distances, are deeply affected by propagation losses. The same conclusions apply to the results in Fig. 17(c), for a fault at $48 \mathrm{~km}$, where the spatial resolution related to the ground mode keeps worsening.

The fact that aerial modes yield a similar resolution for a fault at either 16 and $48 \mathrm{~km}$, is consistent with their negligible propagation losses over $50 \mathrm{~km}$, as expected from (11). This notwithstanding, even restricting transient monitoring to aerialmode contributions, propagation losses would eventually result in a degradation of location accuracy, as soon as they exceed termination losses.

\section{CONCLUSIONS}

This paper has studied the accuracy of fault-location methods based on the computation of similarity between measured full transient signals and reference results. It was shown under what conditions increasing propagation losses may lead to the appearance of spurious fault positions, creating ambiguity in the interpretation of fault-location results, in particular 
when using projection-based metrics rather than correlation. The better performance of correlation was explained by the existence of an additional complementary location mechanism, relying on the decay time of the line resonances.

The first conclusion from this work is that similarity metrics based on projections should be avoided as soon as propagation losses become comparable to dissipation in termination impedances, since they may result in a biased fault location.

As a further practical consequence for lossy single-phase lines, e.g., ground-return overhead lines and coaxial cables, it is not necessarily an effective solution to sample transients at the highest possible rate, since as the maximum frequency increases: a) propagation losses very rapidly increase, frustrating the expected advantage of an improved location accuracy; b) it becomes likelier that tested fault positions close to the probe share some of the resonances of the line under test, planting the seeds for location ambiguity.

Finally, the analysis of a three-phase line has provided some insight in their complex behavior, proving how a single-phaseto-ground fault couples ground and aerial modes, steering the transient propagation to generate section and whole-line resonances. Depending on the relative contributions of ground and aerial modes, spatial resolution was confirmed to significantly change, depending on the fault distance from the probe and the tested bandwidth. The risk of location bias was also confirmed in three-phase lines to be higher for projection-based metrics when propagation losses increase.

These results explain under what conditions fault-location methods based on the similarity between measured and reference transients can be expected to be accurate. Propagation losses have been theoretically predicted, and numerically confirmed, to explain both the loss of resolution and the risk of a biased location. Less intuitively, termination losses were shown to introduce a threshold effect, so that depending on the specific configuration of a line, propagation losses may lead to a varying degree of loss of accuracy.

Future work will focus on the practical implications of these observations, in particular to understand how to take advantage of section and whole-line resonances to improve the fault location accuracy of TBM. As the modal analysis introduced in Sec. VII-B has shown, the nature of the fault has a direct impact on the properties of fault transients. Limitations to the accuracy of fault location might therefore vary across different faults.

\section{APPENDIX}

This appendix discusses how to estimate the fraction of power dissipated within a fault along a three-phase line. Consider a vector of currents $\mathbf{I}^{+}$propagating along the line where the fault is found. The power associated to this excitation is

$$
\begin{aligned}
P_{i} & =\operatorname{Re}\left\{\left(\mathbf{I}^{+}\right)^{\mathrm{H}} \mathbf{V}^{+}\right\}=\left(\mathbf{T} \mathbf{I}_{m}^{+}\right)^{\mathrm{H}}\left(\mathbf{R}_{c} \mathbf{T} \mathbf{I}_{m}^{+}\right) \\
& =\left(\mathbf{I}_{m}^{+}\right)^{\mathrm{H}} \mathbf{T}^{\mathrm{H}} \mathbf{R}_{c} \mathbf{T} \mathbf{I}_{m}^{+}=\left(\mathbf{I}_{m}^{+}\right)^{\mathrm{H}} \mathbf{Q} \mathbf{I}_{m}^{+}
\end{aligned}
$$

where $\mathbf{R}_{c}=\operatorname{Re} \mathbf{Z}_{c}$, while $\mathbf{Q}$ is Hermitian for the line shown in Fig. 11.

Upon its interaction with a fault characterized by a modal reflection matrix $\boldsymbol{\Gamma}_{S}$, a portion $P_{r}$ of the impinging power
$P_{i}$ will be reflected, while a portion $P_{t}$ will be transmitted through the fault and continue propagating along the line.

The reflected power is found similarly to (34)

$$
\begin{aligned}
P_{r} & =\left(\mathbf{I}_{m}^{-}\right)^{\mathrm{H}} \mathbf{V}^{-}=\left(\mathbf{T} \boldsymbol{\Gamma}_{S} \mathbf{I}_{m}^{+}\right)^{\mathrm{H}}\left(\mathbf{R}_{c} \mathbf{T} \boldsymbol{\Gamma}_{S} \mathbf{I}_{m}^{+}\right) \\
& =\left(\mathbf{I}_{m}^{+}\right)^{\mathrm{H}} \boldsymbol{\Gamma}_{S}^{\mathrm{H}} \mathbf{Q} \boldsymbol{\Gamma}_{S} \mathbf{I}_{m}^{+} .
\end{aligned}
$$

The fraction $P_{r} / P_{i}$ of reflected power depends on the relative excitation of the modes, as witnessed by the quadratic form of $P_{i}$ and $P_{r}$. It is nevertheless possible to bound $P_{r} / P_{i}$ by first making a change of base, expressing it as a function of the vector $\mathbf{x}=\mathbf{C I}_{m}^{+}$, instead of $\mathbf{I}_{m}^{+}$, where the matrix $\mathbf{C}$ is the found by means of the Cholesky expansion $\mathbf{Q}=\mathbf{C}^{\mathrm{H}} \mathbf{C}$.

It is now possible to express $P_{r} / P_{i}$ as a Rayleigh quotient

$$
\frac{P_{r}}{P_{i}}=\frac{\mathbf{x}^{\mathrm{H}} \mathbf{K}_{r}^{\mathrm{H}} \mathbf{K}_{r} \mathbf{x}}{\mathbf{x}^{\mathrm{H}} \mathbf{x}}
$$

where $\mathbf{K}_{r}=\mathbf{C} \boldsymbol{\Gamma}_{S} \mathbf{C}^{-1}$. The bounds of Rayleigh quotients are then given by the smallest and largest eigenvalues of the matrix $\mathbf{K}_{r}^{\mathrm{H}} \mathbf{K}_{r}$. Noticing that this matrix is Hermitian, its eigenvalues will necessarily be real and positive.

The fraction of transmitted power $P_{t} / P_{i}$ can be found following the same procedure, substituting $\mathbf{K}_{r}$ with $\mathbf{K}_{t}=$ $\mathbf{C}\left(\mathbf{1}+\boldsymbol{\Gamma}_{S}\right) \mathbf{C}^{-1}$. The fraction of power dissipated by the fault is therefore bounded by

$$
1-\lambda_{0} \leq 1-\frac{P_{r}}{P_{i}}-\frac{P_{t}}{P_{i}} \leq 1-\lambda_{2}
$$

with $\lambda_{0}$ and $\lambda_{2}$ respectively the maximum and minimum eigenvalues of the matrix $\mathbf{K}_{r}^{\mathrm{H}} \mathbf{K}_{r}+\mathbf{K}_{t}^{\mathrm{H}} \mathbf{K}_{t}$.

\section{REFERENCES}

[1] A. Greenwood, Electrical transients in power systems. John Wiley and Sons Inc., New York, USA, 1991.

[2] M. M. Saha, J. J. Izykowski, and E. Rosolowski, Fault location on power networks. Springer Science \& Business Media, 2009.

[3] P. A. Crossley and P. G. McLaren, "Distance protection based on travelling waves," IEEE Trans. Power App. Syst., vol. PAS-102, no. 9, pp. 2971-2983, Sep. 1983.

[4] G. B. Ancell and N. C. Pahalawaththa, "Maximum likelihood estimation of fault location on transmission lines using travelling waves," IEEE Trans. Power Del., vol. 9, no. 2, pp. 680-689, April 1994.

[5] Z. Q. Bo, G. Weller, and M. A. Redfern, "Accurate fault location technique for distribution system using fault-generated high-frequency transient voltage signals," IEE Proceedings - Generation, Transmission and Distribution, vol. 146, no. 1, pp. 73-79, Jan 1999.

[6] Y. Kwon, S. Kang, D. Lee, and H. Kim, "Fault location algorithm based on cross correlation method for HVDC cable lines," in 2008 IET 9th International Conference on Developments in Power System Protection (DPSP 2008), March 2008, pp. 360-364.

[7] O. M. K. K. Nanayakkara, A. D. Rajapakse, and R. Wachal, "Location of DC line faults in conventional HVDC systems with segments of cables and overhead lines using terminal measurements," IEEE Trans. Power Del., vol. 27, no. 1, pp. 279-288, Jan 2012.

[8] Q. Lin, G. Luo, and J. He, "Travelling-wave-based method for fault location in multi-terminal DC networks," The Journal of Engineering, vol. 2017, no. 13, pp. 2314-2318, 2017.

[9] F. V. Lopes, K. M. Silva, F. B. Costa, W. L. A. Neves, and D. Fernandes, "Real-time traveling-wave-based fault location using two-terminal unsynchronized data," IEEE Trans. Power Del., vol. 30, no. 3, pp. 10671076, June 2015.

[10] R. Benato, S. D. Sessa, M. Poli, C. Quaciari, and G. Rinzo, "An online travelling wave fault location method for unearthed-operated high-voltage overhead line grids," IEEE Trans. Power Del., vol. 33, no. 6, pp. 2776-2785, Dec 2018.

[11] R. J. Hamidi and H. Livani, "Traveling-wave-based fault-location algorithm for hybrid multiterminal circuits," IEEE Trans. Power Del., vol. 32, no. 1, pp. 135-144, Feb 2017. 
[12] M. Aurangzeb, P. A. Crossley, and P. Gale, "Fault location on a transmission line using high frequency travelling waves measured at a single line end," in 2000 IEEE Power Engineering Society Winter Meeting. Conference Proceedings (Cat. No.00CH37077), vol. 4, Jan 2000, pp. 2437-2442 vol.4.

[13] M. Farshad and J. Sadeh, "A novel fault-location method for HVDC transmission lines based on similarity measure of voltage signals," IEEE Trans. Power Del., vol. 28, no. 4, pp. 2483-2490, Oct 2013.

[14] F. Deng, X. Li, and X. Zeng, "Single-ended travelling wave protection algorithm based on full waveform in the time and frequency domains," IET Gener. Transm. Distrib, vol. 12, no. 15, pp. 3680-3691, 2018.

[15] R. Razzaghi, G. Lugrin, H. Manesh, C. Romero, M. Paolone, and F. Rachidi, "An efficient method based on the electromagnetic time reversal to locate faults in power networks," IEEE Trans. Power Del., vol. 28, no. 3, pp. 1663-1673, 2013.

[16] S. He, A. Cozza, and Y. Xie, "Electromagnetic time reversal as a correlation estimator: Improved metrics and design criteria for fault location in power grids," IEEE Trans. Electromagn. Compat., in press.

[17] Z. Wang, R. Razzaghi, M. Paolone, and F. Rachidi, "Time reversal applied to fault location in power networks: Pilot test results and analyses," International Journal of Electrical Power \& Energy Systems, vol. 114, p. 105382, 2020.

[18] G. W. Swift, "The spectra of fault-induced transients," IEEE Trans. Power App. Syst., vol. PAS-98, no. 3, pp. 940-947, May 1979.

[19] A. Borghetti, M. Bosetti, C. A. Nucci, M. Paolone, and A. Abur, "Integrated use of time-frequency wavelet decompositions for fault location in distribution networks: Theory and experimental validation," IEEE Trans. Power Del., vol. 25, no. 4, pp. 3139-3146, Oct 2010.

[20] L. U. Iurinic, R. G. Ferraz, and A. S. Bretas, "Characteristic frequency of travelling waves applied for transmission lines fault location estimation," in 2013 IEEE Grenoble Conference, June 2013, pp. 1-5.

[21] Y. Wang, Z. Hao, B. Zhang, and F. Kong, "A pilot protection scheme for transmission lines in VSC-HVDC grid based on similarity measure of traveling waves," IEEE Access, vol. 7, pp. 7147-7158, 2019.

[22] R. Razzaghi, M. Paolone, F. Rachidi, J. Descloux, B. Raison, and N. Retière, "Fault location in multi-terminal HVDC networks based on electromagnetic time reversal with limited time reversal window," in 2014 Power Systems Computation Conference. IEEE, 2014, pp. 1-7.

[23] X. Zhang, N. Tai, Y. Wang, and J. Liu, "EMTR-based fault location for DC line in VSC-MTDC system using high-frequency currents," IET Generation, Transmission Distribution, vol. 11, no. 10, pp. 2499-2507, 2017.

[24] S. He, A. Cozza, and Y. Xie, "On the spatial resolution of fault location techniques based on full fault transients," IEEE Trans. Power Del., 2019, in press.

[25] R. Razzaghi, G. Lugrin, F. Rachidi, and M. Paolone, "Assessment of the influence of losses on the performance of the electromagnetic time reversal fault location method," IEEE Trans. Power Del., vol. 32, no. 5, pp. 2303-2312, Oct 2017.

[26] T. Gonen, Electrical power transmission system engineering: analysis and design. CRC press, 2015.

[27] C. R. Paul, Analysis of multiconductor transmission lines. John Wiley \& Sons, 2008

[28] M. D'Amore and M. S. Sarto, "A new formulation of lossy ground return parameters for transient analysis of multiconductor dissipative lines," IEEE Trans. Power Del., vol. 12, no. 1, pp. 303-314, Jan 1997. 